\title{
Mechanisms of daughter cell-size control during cell division
}

Tomomi Kiyomitsu ${ }^{1,2}$

'Division of Biological Science, Graduate School of Science, Nagoya University Chikusa-ku, Nagoya 464-8602, Japan

${ }^{2}$ Precursory Research for Embryonic Science and Technology (PRESTO) Program, Japan Science and Technology Agency, 4-1-8 Honcho Kawaguchi, Saitama 332-0012, Japan.

Phone: +81-52-788-6174

Fax: +81-52-788-6174

E-mail: kiyomitsu@bio.nagoya-u.ac.jp

Keywords: Spindle positioning, daughter cell size, dynein, myosin, Ran-GTP 


\begin{abstract}
Daughter cell size is tightly regulated during cell division. In animal cells, the position of the anaphase spindle specifies the cell cleavage site to dictate the relative size of the daughter cells. Although spindle orientation is regulated by dynein-dependent cortical pulling forces exerted on astral microtubules in many cell types, it was unclear how these forces are precisely regulated to center or displace the spindle. Recently, intrinsic signals derived from chromosomes or spindle poles have been demonstrated to regulate dynein-dependent pulling forces in symmetrically dividing cells. Unexpectedly, myosin-dependent contractile forces have also been shown to control spindle position by altering the cellular boundaries during anaphase. In this review, I discuss how dyneinand myosin-dependent forces are coordinately regulated to control daughter cell size.
\end{abstract}

123 words 


\section{Daughter cell size is defined by anaphase spindle position}

Cell division is the fundamental process by which two daughter cells are produced from a single progenitor. In animal cells, the site of cell cleavage to "cut" the cell in two is specified during anaphase [1-3] by signals derived from the spindle midzone, which is formed between the separating chromosomes (Fig. 1a, [4]). This mechanism coordinates chromosome segregation with cytokinesis and ensures the equal distribution of the duplicated chromosomes into daughter cells to maintain the genomic information. In contrast, other factors such as polarized cell fate determinants and extrinsic niche signals can be symmetrically or asymmetrically distributed between daughters by controlling spindle orientation (Fig. 1a, see left and center, and; Fig. 1b, reviewed in [5]). Because the distribution of these polarized factors is critical to determine the fate of the daughter cells, and thus the cell division type (symmetric or asymmetric cell division) [6], the mechanisms of spindle orientation have been extensively studied in several organisms during development [7-13] and in vitro cell culture [14]. These studies have established a key concept that polarity signals assemble force-generating machineries at specific sites at the cell cortex to properly orient the spindle along the polarity axis [5].

Similar to the way in which the distribution of polarity factors can be controlled by modulating spindle orientation, the physical size of the daughter cells is varied by controlling spindle position (Fig. 1a, center and right). However, compared to the detailed understanding that exists for the basis of spindle orientation, the mechanisms and roles of spindle positioning and daughter cell-size control were poorly understood. In most vertebrate cells, the spindle is positioned in the center of the cell resulting in equal-sized cell division (Fig. 1b I and III). In contrast, the spindle is displaced to generate unequal-sized daughters in several different cell types, such as the $C$. elegans early embryo [8], Drosophila neuroblasts [7], sea urchin blastomeres [2], and mouse oocytes [15-17]. These unequally dividing daughters display different cell fates due to both differences in cell size, as well as asymmetric distribution of cell-fate determinants as a result of controlled spindle orientation (Fig. 1b IV). However, a difference in cell size is not always required for asymmetric cell fate during asymmetric cell division (Fig. 1b III), and the contribution of the relative daughter 
cell size to defining cell fate was unclear.

Nonetheless, daughter cell size appears to be tightly regulated during both symmetric and asymmetric cell division. Indeed, the difference in cell volume or mass between two daughter cells in symmetrically dividing L1210 lymphoblasts is less than $10 \%[18,19]$. Daughter cell size affects the relative amount of cytoplasmic factors and organelles, such as mitochondria, which are critical for cell function and survival [20]. Furthermore, daughter cell size may have critical roles in early interphase events, such as nucleus formation, and may affect the relative volume of the nucleus and cytoplasm, which is maintained as a constant ratio during cell cycle [21]. Thus, it is plausible that daughter cell-size control plays a critical role in cell physiology. Recent studies indicate that altering daughter cell size results in differential behavior of daughter cells in both symmetrically and asymmetrically dividing cells [22-24].

To understand the mechanisms of spindle positioning, it is critical to define the forces acting on the spindle to control its position. Accumulating evidence indicates that the minus-end directed microtubule-based motor cytoplasmic dynein $[25,26]$ (hereafter called dynein), generates cortical pulling forces that act on astral microtubules to control spindle orientation and position $[8,27,28]$. However, the mechanisms that precisely regulate cortical dynein localization and activity to control spindle position is poorly understood, especially in symmetrically dividing cells. Here, I review the recent advances in understanding how dynein-dependent cortical pulling forces are regulated during metaphase and anaphase to center or displace the spindle, thus generating equal or unequal-sized daughters, respectively.

Direct contacts between astral microtubules and the cell cortex act to generate a physical connection and generate force to move the spindle in most cell types. However, in extremely large cells, such as Xenopus zygotes [29], and in cells with anastral spindles such as mouse oocytes [15-17] or plant cells [12], astral microtubules do not reach the cell cortex. In these cells, spindle position must be regulated by different mechanisms such as length-dependent astral microtubule pulling in the cytoplasm [29-32] or actin-based forces both in the cytoplasm [16, 33] and at the cell cortex [34]. As these mechanisms have been reviewed extensively $[15,17,35,36]$, I will instead focus on the mechanisms of 
spindle positioning by cortical pulling forces during metaphase and anaphase.

\section{Cortical dynein recruitment in unequally dividing cells}

Over the past few decades, the mechanisms of spindle displacement and the resulting unequal-sized cell division have been extensively studied in the asymmetrically dividing one-cell $C$. elegans embryo (reviewed in detail in $[8,28]$ ). In these cells, the maternal and paternal pronuclei and their associated centrosomes are initially positioned in the center of the cell during interphase by microtubule-length dependent pulling mechanisms [31]. Subsequently, the spindle forms and is displaced toward the posterior cell cortex during anaphase (Fig. 2a). Dynein is a key player in the generation of asymmetric pulling forces that act on astral microtubules $[8,28]$. In $C$. elegans embryos, a conserved cortical dynein receptor (GPR-1/2) asymmetrically accumulates at the posterior cell cortex, downstream of polarity signals. GRP-1/2 forms a ternary complex with membrane-bound $\mathrm{G} \alpha$ proteins (GOA-1 and GPA-16) and LIN-5, which are likely to recruit or activate dynein at the posterior cell cortex [37, 38]. Although the asymmetric enrichment of dynein is not detectable in these one-cell embryos, over-expression of GPR-1 causes the asymmetric cortical enrichment of dynein at the posterior cell cortex [39]. During the second cell division in these embryos, there is a clear cortical enrichment of dynein that is dependent on cortical LIN-5, GPR-1/2, and Ga that is observed even without overexpression of GPR-1 [38]. Thus, current models suggest that cortical dynein is asymmetrically recruited and/or activated at the cell cortex via a LIN-5-GPR-1/2-G $\alpha$ ternary complex to displace the spindle in unequally dividing $C$. elegans embryos (Fig. 2a), although an additional $\mathrm{G} \alpha$-independent mechanism for cortical force generation is also proposed [40].

\section{Cortical dynein recruitment in symmetrically dividing HeLa cells}

The regulation of cortical dynein localization and activity observed in $C$. elegans provides a generalizable paradigm for motor-driven spindle movement. The ternary complex containing LIN-5, GPR-1/2, and Ga (GOA-1, GPA-16) that is present in $C$. elegans is conserved in Drosophila (Mud/Pins/G $\alpha$ ) and vertebrates (NuMA/LGN/Gai) [5]. Dynein is also required for spindle orientation in budding 
yeast, but in this case Num1 functions as a cortical dynein receptor [41]. Among the subunits of the ternary complex, NuMA has been implicated as a direct binding partner for dynein or its associated proteins, as the $\mathrm{N}$-terminal region of NuMA is sufficient to recruit the dynein-dynactin complex to the cell cortex in symmetrically dividing human HeLa cells [39].

Whereas the ternary NuMA-LGN-Gai complex represents the lone pathway for cortical dynein recruitment in metaphase HeLa cells [42], NuMA and the dynein-dynactin complex can be targeted to the cell cortex independently of LGN and Gai during anaphase [22, 43-45] (Fig. 2b). This anaphase-specific recruitment of dynein to the cell cortex is negatively regulated in metaphase by CDK-dependent phosphorylation of the C-terminal region of NuMA at T2055 [22, $46,47]$, and is activated by dephosphorylation via PPP2CA during anaphase [47]. Two different molecules have been proposed to act as cortical receptors for dephosphorylated NuMA in the anaphase-specific pathway (Fig. 2b). One candidate involves the conserved family of 4.1 proteins [22]. Vertebrates contain 4 isoforms of 4.1 , referred to as 4.1R/EPB41, 4.1N/EPB41L1, 4.1G/EPB41L2, and $4.1 \mathrm{~B} / \mathrm{EPB} 41 \mathrm{~L} 3$. These 4.1 proteins act as hubs to organize membrane proteins through several domains including FERM and spectrin/actin-binding domains, and a unique C-terminal domain (CTD) [48]. The conserved C-terminal domain of 4.1 (4.1-CTD) interacts directly with the C-terminal region of NuMA [49], and is necessary and sufficient for the cortical targeting of NuMA and dynein during anaphase [22]. Although the precise mode of interaction between the 4.1-CTD and NuMA during anaphase is still unclear, phosphorylated NuMA shows reduced interactions with the 4.1-CTD [22]. A second candidate for recruiting NuMA to the cell cortex during anaphase is phosphoinositides (PIPs), which interact with the C-terminal region of NuMA [43, 45]. At present, two separate regions of NuMA have been proposed as direct binding sites for PIPs. One of these contains the CDK-phosphorylation site (T2055 residue) [43], suggesting that CDK-dependent phosphorylation may prevent NuMA from interacting with phosphoinositides directly by introducing a negative charge. How the other potential PIP-binding region on NuMA is regulated by T2055 phosphorylation remains unclear.

If cortical dynein is widely used as a force generator to control spindle 
position or orientation, additional cortical dynein recruitment pathways may exist during asymmetric cell division to respond to different polarity signals. In fact, the LGN-binding partner, Inscuteable, is expressed in asymmetrically dividing mouse epidermal progenitor cells [50] and functions to recruit LGN to the cell cortex by linking LGN with the Par polarity complex [11]. Similarly, Mud/NuMA interacts with Dishevelled, which links Mud/NuMA to the transmembrane receptor Frizzled in Drosophila sensory organ precursors [51]. These additional pathways that recruit cortical dynein receptors likely provide distinct and alternative ways of generating cortical pulling forces during asymmetric cell division [5].

\section{Cortical dynein regulation and spindle centering in metaphase}

During cell division, most animal cells have a round shape, which ensures proper space for chromosome alignment and spindle assembly [52], and facilitates spindle positioning [53]. Since the spindle is not precisely positioned during prometaphase, dynein-dependent cortical pulling forces must act to properly control spindle position during metaphase and anaphase. Recent studies have demonstrated that chromosome- and spindle pole-derived signals regulate cortical dynein recruitment during metaphase in symmetrically dividing HeLa cells [42]. Chromosome-derived Ran-GTP gradients negatively regulate the interaction between Gai and LGN-NuMA in the vicinity of the metaphase chromosomes (Fig. 3). This helps to restrict cortical dynein to the polar cell cortex, downstream of NuMA-LGN, and maintain the spindle orientation axis [42]. Although the precise molecular mechanisms by which Ran-GTP dissociates NuMA-LGN from Gai remain unclear, NuMA is an excellent target for Ran-GTP regulation, as NuMA is an established downstream target of the Ran gradient that directs spindle assembly [54, 55].

To align the spindle in the center of the cell, cortical pulling force must be properly regulated to rectify spindle mis-positioning. Importantly, Polo-like kinase 1 (Plk1) signals derived from the spindle pole negatively regulate the interaction between the NuMA-LGN complex and dynein-dynactin in a distance-dependent manner [42] (Fig. 3). This mechanism autonomously generates cortical dynein asymmetry when the spindle is displaced from the cell center, resulting in the 
generation of asymmetric cortical pulling forces to correct spindle mis-positioning [42] (Fig. 3). Since NuMA and LGN occasionally display asymmetric localization in symmetrically dividing cells $([56,57]$, T.K. unpublished observation), the Ran-GTP system may also negatively regulate NuMA-LGN localization at the polar cell cortex to facilitate spindle centering, especially when the spindle is highly displaced from the cell center.

Together, these Ran- and Plk1-dependent negative feedback systems control dynein-dependent cortical pulling forces to achieve spindle centering during metaphase in symmetrically dividing HeLa cells. Such distance-dependent regulation by intracellular gradients are also used in other biological process, such as the correction of kinetochore-microtubule attachment defects by Aurora-B kinase $[58,59]$, cell size homeostasis in fission yeast by the Pom1 kinase [60-62], and division site specification in Caulobacter crescentus by Mip-Z (reviewed in $[63,64]$ ). Intracellular gradients are useful for transmitting positional information to downstream targets and may be widely used for spatial control. While dynein-dependent cortical pulling forces appear to play a dominant role in spindle movement within HeLa cells, other mechanisms such as length-dependent astral microtubule pulling in the cytoplasm [29, 31], astral microtubule buckling [65], spindle length control [66], actin-dependent mechanisms [67], and control of cell size or geometry [53] may also cooperate to center the spindle.

\section{Spindle centering by membrane elongation during anaphase}

During early anaphase, cell shape remains constant and the distribution of cortical dynein is regulated similarly to metaphase, with dynein-dependent asymmetric pulling forces moving the spindle to rectify spindle mis-positioning [22] (Fig. 4a-1). In contrast to the presence of checkpoints that monitor spindle assembly and chromosome-segregation [68], checkpoint systems that inhibit anaphase onset until the spindle is properly positioned appear to be absent, at least in HeLa (human), LLC-PK (pig), and MDCK (dog) cells [22, 57, 69]. Instead, vertebrate cells have developed back-up systems to rectify spindle mis-positioning during anaphase by coordinately controlling spindle movement and cortical membrane elongation [22]. During the later stages of anaphase, cell 
shape changes dynamically, providing an alternative mechanism to rectify spindle mis-positioning by asymmetrically expanding the polar cell cortex and alter the cellular boundaries relative to the spindle (Fig. 4a-2). This asymmetric cortical expansion occurs in response to spindle positioning defects at the end of early anaphase: only the polar membrane that is closest to the spindle elongates, with the other side remaining stationary (Fig. 4a-2). Since cortical expansion is a rapid process (roughly estimated $1.4 \mu \mathrm{m} / \mathrm{min}$ in HeLa cells [22]) as compared to dynein-dependent spindle movement $(0.05-0.40 \mu \mathrm{m} / \mathrm{min}$ in HeLa cells [42]), this may be an ideal way to rectify spindle mis-positioning within a limited time-period during late anaphase. However, rapid cortical expansion results in a flow of cytosol that moves the spindle toward the growing cell cortex [70]. Thus, cortical dynein must act at the stationary cell cortex to anchor the spindle and prevent spindle displacement [22] (Fig. 4a-2, 4b). In the absence of cortical dynein during anaphase, the spindle becomes displaced due to the cytosolic flow caused by asymmetric membrane elongation. This spindle movement results in unequally sized daughter cells following cell division [22]. Thus, the coordinated action of cortical dynein and asymmetric membrane elongation is critical to center the spindle during late anaphase (Fig. 4b).

Asymmetric membrane elongation during anaphase represents a distinct paradigm for controlling spindle position. As local secretion of new membrane from internal vesicles is not observed at the growing cell cortex [22], cortical remodeling of the pre-existing cell cortex plays a major role in asymmetric cortical expansion. During anaphase, cortical rigidity is largely regulated by actomyosin-based contractile forces acting on the plasma membrane [4]. While actomyosin accumulates at the equatorial region downstream of midzone signals to form a contractile ring for cytokinesis, a substantial amount of actin and myosin remain at the polar cell cortex during anaphase [70]. When the polar actomyosin levels are equal on both sides, the polar membrane expands symmetrically as the cleavage furrow ingresses (Fig. 4c, symmetric). In contrast, when polar actomyosin is asymmetric, unbalanced forces in the polar cell cortex can trigger cell shape instabilities or asymmetric cortical expansion [71] (Fig. 3c, asymmetric). In this case, the myosin-rich cortex contracts, whereas the opposing polar cell cortex with reduced myosin asymmetrically expands to alter 
the cellular boundaries [22, 24, 72] (Fig. 3c, asymmetric). Similarly, cortical remodeling by membrane-actin linkers, such as moesin, at the polar cell cortex regulates polar relaxation during anaphase $[73,74]$. Thus, the asymmetry of the cortical factors that regulate contraction or relaxation of the polar cell cortex can drive asymmetric cortical membrane elongation during anaphase.

These components of the actin cytoskeleton and cell cortex provide the molecular players to achieve the physical expansion of the plasma membrane to control spindle position. However, the question remains as to what signals generate the polar asymmetry of these cortical factors in response to spindle mis-positioning during anaphase. A possible candidate is the chromosome-derived Ran-GTP gradient, as it inhibits the localization of the cortical scaffold protein, anillin [75], and its downstream target myosin II at the growing cell cortex [22] (Fig. 4b). Although the precise mechanisms are still unclear, anillin is a good candidate for a target of Ran-GTP as it contains a nuclear localization sequence (NLS) and is regulated by Ran-GTP during pseudocleavage formation in Drosophila syncytial embryos [76]. Given the complexity of the cortical structure and its regulation, additional intrinsic signals derived from chromosomes, spindle pole, or astral microtubules are also likely to regulate the localization or activity of anillin and other cortical proteins for cortical expansion [4].

In summary, cortical contractile forces at the polar cell cortex are spatially regulated by intrinsic signals in response to the anaphase spindle position during equal-sized cell division. These mechanisms control whether the polar membrane elongates symmetrically or asymmetrically, which contributes to rectifying spindle mis-positioning by altering the cellular boundaries during equal-sized cell division.

\section{Daughter cell-size control by dynein- and myosin-based asymmetric forces}

Symmetrically dividing cells have developed intrinsic error-correction systems that autonomously generate dynein- or myosin-based asymmetric forces to rectify spindle mis-positioning to generate equal-sized daughters (Fig. 5a). As these systems and their regulators such as Ran, Plk1, and anillin have multiple 
essential roles during cell division [75, 77-79], these components are likely to exist in unequally dividing cells. Thus, another question that arises is how do cells eliminate or modulate these spindle-centering systems to divide unequally?

In unequally dividing $C$. elegans one-cell embryos, the chromosome-derived Ran gradient may not reach the cell cortex due to the relatively long distance between the chromosomes and the polar cell cortex (more than $10 \mu \mathrm{m}$ ). Although the size of the Ran-GTP gradient is likely to differ between cell types [80], the working distance is approximately 2 and $10 \mu \mathrm{m}$ in HeLa cells and mouse oocytes, respectively [42, 81]. Interestingly, Polo-like kinase 1 (PLK-1) is distributed asymmetrically in the anterior cytoplasm downstream of Par polarity signals [82, 83], which may neutralize opposing pulling forces for spindle centering. In addition, due to the nature of the eggshell in these embryos, which provides a fixed external barrier, drastic asymmetric membrane elongation does not occur at the posterior cell cortex, even if the spindle comes close to the posterior cell cortex. These mechanisms would coordinately promote spindle displacement, although some buffering mechanisms appears to exist so as to not displace the spindle all the way to one side of the cell and generate daughter cells that are too small [28].

As the $C$. elegans one-cell embryo is a relatively large cell, different mechanisms may exist in smaller cells for spindle displacement. Recent studies in C. elegans neuroblasts, which are much smaller than the one-cell embryo, revealed that cortical myosin asymmetry is generated during anaphase and drives unequal-sized cell division by asymmetrically elongating the polar cell cortex [24] (Fig. 5b). Similar asymmetric cortical expansion has also been reported in Drosophila neuroblasts [72]. Contrary to symmetrically dividing cells, this cortical myosin asymmetry is likely to be generated independently of the chromosome/spindle-derived signals, but does depend on polarity signals [84].

Given that the Drosophila dynein receptor, Pins (the LGN ortholog) localizes asymmetrically to the growing apical cell cortex downstream of Par proteins [85-87], it is unclear how the spindle can counteract cytosol flow, which is likely to be generated from the basal to apical cell cortex during asymmetric membrane elongation. As Drosophila neuroblasts generate much larger apical centrosomes, and thus longer astral microtubules at the apical side [23, 88, 89], 
this spindle asymmetry may function to counteract the cytosol flow and displace the spindle toward the basal side during asymmetric cortical expansion (Fig. 5b). DSas4-mutant flies, which lack astral microtubules, display partial defects in daughter cell-size asymmetry in neuroblasts [90], indicating that asymmetrically longer astral microtubules are likely to contribute to displacing the spindle. Apical complex genes and $\mathrm{G} \beta / \gamma$ signals also contribute to control daughter cell-size asymmetry in Drosophila neuroblasts [23, 88, 89, 91], although the precise mechanisms are still unclear.

Polarity signals must have a key role in generating asymmetric forces, which dominantly function to displace the spindle and/or inhibit spindle-centering mechanisms in unequally dividing cells. In cases where the polarity signals generate cortical pulling forces, but do not inhibit spindle-centering systems, the signals may generate "torque" to orient the spindle, but not displace the spindle away from the cell center, resulting in properly oriented and equally sized daughter cells (Fig. 1b-III).

\section{Concluding remarks}

Compared with the current understanding of spindle orientation, the mechanisms of spindle positioning remain poorly understood. However, recent studies have revealed key players and a basic framework for spindle position control, which defines daughter cell size during cell division. The cortical force-generating machinery that acts on astral microtubules is the primary factor required to control spindle orientation. In contrast, cortical forces derived from both dynein-dependent cortical pulling forces acting on astral microtubules and myosin-dependent contractile forces acting at the polar cell cortex act in coordination to control spindle position by moving the spindle or altering cellular boundaries, respectively (Fig. 5).

Intrinsic signals derived from the chromosomes or the spindle autonomously create cortical dynein and myosin asymmetries within the cell in response to spindle mis-positioning. These signals control spindle movement or membrane elongation to center the spindle during anaphase and ensure that cells divide equally (Fig. 5a). In contrast, to divide unequally, polarity signals create cortical asymmetry of these motor proteins within the cell and displace the 
spindle by asymmetrically moving the spindle or altering cellular boundaries (Fig. $5 b$ ). Defining the precise molecular mechanisms of asymmetric force generation downstream of intrinsic signals and polarity signals, respectively, is critical to understand daughter cell-size control. In particular, how polarity signals disrupt or modulate intrinsic spindle centering systems are important future questions, as Ran, Plk1, and their downstream targets appear to be differently regulated between equally dividing HeLa cells [42] and unequally dividing $C$. elegans embryos [82, 83] or mouse oocytes [81]. In addition to polarity signals, daughter cell size is affected by cell geometry $[2,35,53]$ and cell adhesion [18], which are variable between cell types and during development. Understanding the mechanisms by which these factors affect the spindle positioning systems described here is an important open question.

As both kinetochores and the cell cortex function as attachment sites for a microtubule plus-ends, the strategies, techniques and concepts from the kinetochore field [92] are useful to understand mechanisms that specify, assemble, and regulate force-generating machineries at the cell cortex. For example, a proteomics-based approach would be useful to identify binding partners of key cortical proteins. In addition, in vitro reconstitution of astral microtubule-cell cortex interaction would provide useful information to understand the detailed mechanics of force generation and spindle positioning. Centrosome positioning was recently reconstituted in vitro using purified centrosomes and yeast dynein combined within a micro-chamber [93]. This study revealed that dynein induces microtubule catastrophe and generates force by interacting with depolymerizing microtubules, analogous to the Ndc80/Ska1 complex at kinetochores [94]. As dynein is an approximately 1.4 MDa multi-protein complex, it has long been difficult to reconstitute dynein holo-complex in vitro. However, the human dynein holo-complex was recently reconstituted in vitro [95], which revealed that the ternary complex formation of dynein, dynactin and adaptor proteins such as Bicaudal $D$ is required for the activation of human dynein motility [96, 97]. In addition, other dynein-binding partners such as Lis1 and NudE regulate the force producing state and/or the prolonged microtubule attachment of dynein [98, 99]. Investigating the mechanisms by which these dynein-binding partners regulate microtubule 
capture, astral microtubule dynamics, and force generation in vitro would provide important mechanistic insights to understand spindle positioning.

Although the relationship between spindle orientation and cell-fate determination has been established, the functional link between daughter cell size and daughter cell fate remains largely unknown in animal cells. To understand this, it is critical to manipulate daughter cell size without affecting other polarized cell-fate determinants. Recently, unequal-sized daughters have been generated in cells, which normally divide symmetrically (Fig. 1b-II), using genetics [22], artificially controlling cell geometry [2, 53], or by directly manipulating spindle position [100]. Analyzing the behavior of resulting smallerand larger-sized daughters of different cell types could provide additional information to understand the role of daughter cell-size control. Defining the mechanisms and roles of daughter cell-size control in both symmetrically and asymmetrically dividing cells will continue to be an exciting area of future research.

3995 words 


\section{Figure legends}

Figure 1. Daughter cell-size control by spindle positioning (a) Spindle orientation controls the distribution of polarized factors (compare Left with Center), whereas spindle position defines daughter cell size (compare Center to Right). Left: during symmetric cell division, the spindle is centrally positioned and all cellular components are equally distributed between daughter cells. Center: during asymmetric cell division in most vertebrate cells, the spindle is oriented parallel to the polarity axis to asymmetrically distribute polarized factors, whereas the spindle is positioned in the center of the cell to divide equally in size. Right: in a subset of asymmetrically dividing cells, the spindle is displaced and both, daughter cell size and the distribution of polarized factors become asymmetric. The blue line and green circle indicate chromosomes and centrosomes, respectively. (b) Cell division can be classified into 4 groups in terms of daughter cell size and distribution of polarized factors.

Fig. 2 Cortical dynein recruitment pathways (a) A model showing recruitment of cortical dynein by the conserved ternary complex, LIN-5, GPR-1/2, and $G \alpha$ proteins (GOA-1 and GPA-16), in unequally dividing C. elegans embryos. (b) Diagram showing two parallel pathways that target dynein and dynactin at the cell cortex during metaphase and anaphase in symmetrically dividing HeLa cells. NuMA-LGN-Gai recruits dynein during metaphase and anaphase, whereas 4.1and/or PIPs- NuMA pathways targets dynein specifically during anaphase [22, 43-45].

Fig. 3 Spindle centering systems in metaphase A model showing spindle centering systems in metaphase. Chromosome-derived Ran-GTP gradient (blue) negatively regulates the interaction between Gai and LGN-NuMA and excludes LGN-NuMA from the cell cortex near chromosomes. This system confines LGN, NuMA, and dynein at the polar cell cortex and contributes to maintain the axis for spindle orientation. In contrast, spindle-pole derived Plk1 signals (red) negatively control the interaction between LGN-NuMA and dynein in a distance-dependent manner. This system autonomously regulates cortical 
dynein localization in response to spindle mis-positioning and generates asymmetric pulling force to correct spindle mis-positioning.

Fig. 4 Spindle centering systems in anaphase (a) Diagrams summarizing anaphase spindle centering processes. When the spindle is centrally positioned by the end of metaphase, the central spindle position is maintained during anaphase and the polar membrane elongates symmetrically. 1) Spindle mis-positioning is rectified by moving the spindle during early anaphase. Once the spindle is centrally positioned, this is maintained until cytokinesis. 2) When the spindle is mis-positioned at the end of early anaphase, cells autonomously generate cortical dynein and myosin asymmetry in response to the spindle position. Myosin asymmetry causes asymmetrical membrane elongation, whereas dynein asymmetry produces cortical pulling forces to move or anchor the spindle at the stationary cell cortex. 3) When the spindle is fully elongated during anaphase to reach the polar cell cortex, the spindle is naturally placed in the center of the cell. (b) Diagram showing regulation of asymmetrical membrane elongation during anaphase. Ran-GTP functions to initiate the local reduction of polar anillin and myosin at the growing polar cell cortex near the chromosomes. Anillin and myosin actively accumulate at the equatorial cell cortex by midzone-derived signals, which negatively regulate cortical NuMA localization at the equatorial cell cortex during anaphase. (c) When the polar actomyosin level (magenta) is identical on both sides, the polar cell cortex expands symmetrically as the equatorial cell cortex (gray) ingresses during anaphase. In contrast, polar region asymmetrically extends when polar actomyosin level is asymmetrical. Asymmetric cortical contraction causes cytosolic flow (light gray arrows) toward the growing cell cortex (light green) and asymmetrically expands the cellular boundary.

Fig. 5 Mechanisms of equal- and unequal-sized cell division (a) During equal-sized cell division, cortical dynein and polar myosin asymmetry are generated in response to spindle mis-positioning downstream of intrinsic signals. Dynein and myosin asymmetry generates asymmetrical pulling forces that move the spindle and asymmetrical contractile forces that alter cellular boundary, 
respectively. These asymmetrical forces are balanced once the spindle is positioned in the center of the cell. (b) During unequally sized cell division, cortical dynein and polar myosin asymmetry are generated by polarity signals. Similarly, in equally sized cell division dynein and myosin asymmetry generates asymmetrical pulling forces and asymmetric contractile forces, respectively, but displace the spindle. In addition, these asymmetric forces are not balanced during anaphase, resulting in unequally sized cell division. 


\section{Acknowledgments}

I thank lain Cheeseman, Akatsuki Kimura, Gohta Goshima, Sadanori Watanabe, and Shigeki Yoshiura for critical comments and suggestions. I also thank Danielle Loughlin and reviewers for helpful comments. The author's research is supported by JST, PRESTO program and JSPS KAKENHI (grant number 26840063). T.K is a recipient of a research grant from the NOVARTIS Foundation (Japan) for the Promotion of Science, and a Career Development Award of Human Frontier Science Program.

\section{References (80-100)}

1. Rappaport, R. (1985). Repeated furrow formation from a single mitotic apparatus in cylindrical sand dollar eggs. The Journal of experimental zoology 234, 167-171.

2. Rappaport, R. (1996). Cytokinesis in animal cells. Cambridge university press.

3. Burgess, D.R., and Chang, F. (2005). Site selection for the cleavage furrow at cytokinesis. Trends Cell Biol 15, 156-162.

4. Green, R.A., Paluch, E., and Oegema, K. (2012). Cytokinesis in animal cells. Annu Rev Cell Dev Biol 28, 29-58.

5. Morin, X., and Bellaiche, Y. (2011). Mitotic spindle orientation in asymmetric and symmetric cell divisions during animal development. Dev Cell 21, 102-119.

6. Horvitz, H.R., and Herskowitz, I. (1992). Mechanisms of asymmetric cell division: two Bs or not two Bs, that is the question. Cell 68, 237-255.

7. Siller, K.H., and Doe, C.Q. (2009). Spindle orientation during asymmetric cell division. Nat Cell Biol 11, 365-374.

8. Gonczy, P. (2008). Mechanisms of asymmetric cell division: flies and worms pave the way. Nat Rev Mol Cell Biol 9, 355-366.

9. Knoblich, J.A. (2008). Mechanisms of asymmetric stem cell division. Cell 132, 583-597.

10. Inaba, M., and Yamashita, Y.M. (2012). Asymmetric stem cell division: precision for robustness. Cell Stem Cell 11, 461-469. 
11. Williams, S.E., and Fuchs, E. (2013). Oriented divisions, fate decisions. Curr Opin Cell Biol 25, 749-758.

12. Rasmussen, C.G., Humphries, J.A., and Smith, L.G. (2011). Determination of symmetric and asymmetric division planes in plant cells. Annu Rev Plant Biol 62, 387-409.

13. Shitamukai, A., and Matsuzaki, F. (2012). Control of asymmetric cell division of mammalian neural progenitors. Dev Growth Differ 54, 277-286.

14. Habib, S.J., Chen, B.C., Tsai, F.C., Anastassiadis, K., Meyer, T., Betzig, E., and Nusse, R. (2013). A localized Wnt signal orients asymmetric stem cell division in vitro. Science 339, 1445-1448.

15. Almonacid, M., Terret, M.E., and Verlhac, M.H. (2014). Actin-based spindle positioning: new insights from female gametes. J Cell Sci 127, 477-483.

16. Holubcova, Z., Howard, G., and Schuh, M. (2013). Vesicles modulate an actin network for asymmetric spindle positioning. Nat Cell Biol 15, 937-947.

17. Li, R., and Albertini, D.F. (2013). The road to maturation: somatic cell interaction and self-organization of the mammalian oocyte. Nat Rev Mol Cell Biol 14, 141-152.

18. Sung, Y., Tzur, A., Oh, S., Choi, W., Li, V., Dasari, R.R., Yaqoob, Z., and Kirschner, M.W. (2013). Size homeostasis in adherent cells studied by synthetic phase microscopy. Proc Natl Acad Sci U S A 110, 16687-16692.

19. Tzur, A., Kafri, R., LeBleu, V.S., Lahav, G., and Kirschner, M.W. (2009). Cell growth and size homeostasis in proliferating animal cells. Science 325, 167-171.

20. Dalton, C.M., and Carroll, J. (2013). Biased inheritance of mitochondria during asymmetric cell division in the mouse oocyte. J Cell Sci 126, 2955-2964.

21. Hara, Y., and Kimura, A. (2011). Cell-size-dependent control of organelle sizes during development. Results and problems in cell differentiation 53 , 93-108.

22. Kiyomitsu, T., and Cheeseman, I.M. (2013). Cortical dynein and asymmetric membrane elongation coordinately position the spindle in 
anaphase. Cell 154, 391-402.

23. Fuse, N., Hisata, K., Katzen, A.L., and Matsuzaki, F. (2003). Heterotrimeric $\mathrm{G}$ proteins regulate daughter cell size asymmetry in Drosophila neuroblast divisions. Curr Biol 13, 947-954.

24. Ou, G., Stuurman, N., D'Ambrosio, M., and Vale, R.D. (2010). Polarized myosin produces unequal-size daughters during asymmetric cell division. Science 330, 677-680.

25. Kardon, J.R., and Vale, R.D. (2009). Regulators of the cytoplasmic dynein motor. Nat Rev Mol Cell Biol 10, 854-865.

26. Roberts, A.J., Kon, T., Knight, P.J., Sutoh, K., and Burgess, S.A. (2013). Functions and mechanics of dynein motor proteins. Nat Rev Mol Cell Biol 14, 713-726.

27. Grill, S.W., and Hyman, A.A. (2005). Spindle positioning by cortical pulling forces. Dev Cell 8, 461-465.

28. McNally, F.J. (2013). Mechanisms of spindle positioning. J Cell Biol 200, 131-140.

29. Wuhr, M., Tan, E.S., Parker, S.K., Detrich, H.W., 3rd, and Mitchison, T.J. (2010). A model for cleavage plane determination in early amphibian and fish embryos. Curr Biol 20, 2040-2045.

30. Kimura, A., and Onami, S. (2005). Computer simulations and image processing reveal length-dependent pulling force as the primary mechanism for C. elegans male pronuclear migration. Dev Cell 8, 765-775.

31. Kimura, K., and Kimura, A. (2011). Intracellular organelles mediate cytoplasmic pulling force for centrosome centration in the Caenorhabditis elegans early embryo. Proc Natl Acad Sci U S A 108, 137-142.

32. Minc, N., Burgess, D., and Chang, F. (2011). Influence of cell geometry on division-plane positioning. Cell 144, 414-426.

33. Yi, K., Rubinstein, B., Unruh, J.R., Guo, F., Slaughter, B.D., and Li, R. (2013). Sequential actin-based pushing forces drive meiosis I chromosome migration and symmetry breaking in oocytes. J Cell Biol 200, 567-576.

34. Chaigne, A., Campillo, C., Gov, N.S., Voituriez, R., Azoury, J., 
Umana-Diaz, C., Almonacid, M., Queguiner, I., Nassoy, P., Sykes, C., et al. (2013). A soft cortex is essential for asymmetric spindle positioning in mouse oocytes. Nat Cell Biol 15, 958-966.

35. Minc, N., and Piel, M. (2012). Predicting division plane position and orientation. Trends Cell Biol 22, 193-200.

36. Mitchison, T., Wuhr, M., Nguyen, P., Ishihara, K., Groen, A., and Field, C.M. (2012). Growth, interaction, and positioning of microtubule asters in extremely large vertebrate embryo cells. Cytoskeleton (Hoboken) 69, 738-750.

37. Couwenbergs, C., Labbe, J.C., Goulding, M., Marty, T., Bowerman, B., and Gotta, M. (2007). Heterotrimeric $G$ protein signaling functions with dynein to promote spindle positioning in C. elegans. J Cell Biol 179, 15-22.

38. Nguyen-Ngoc, T., Afshar, K., and Gonczy, P. (2007). Coupling of cortical dynein and $G$ alpha proteins mediates spindle positioning in Caenorhabditis elegans. Nat Cell Biol 9, 1294-1302.

39. Kotak, S., Busso, C., and Gonczy, P. (2012). Cortical dynein is critical for proper spindle positioning in human cells. J Cell Biol 199, 97-110.

40. Hara, Y., and Kimura, A. (2009). Cell-size-dependent spindle elongation in the Caenorhabditis elegans early embryo. Curr Biol 19, 1549-1554.

41. Tang, X., Germain, B.S., and Lee, W.L. (2012). A novel patch assembly domain in Num1 mediates dynein anchoring at the cortex during spindle positioning. J Cell Biol 196, 743-756.

42. Kiyomitsu, T., and Cheeseman, I.M. (2012). Chromosome- and spindle-pole-derived signals generate an intrinsic code for spindle position and orientation. Nat Cell Biol 14, 311-317.

43. Zheng, Z., Wan, Q., Meixiong, G., and Du, Q. (2014). Cell cycle-regulated membrane binding of NuMA contributes to efficient anaphase chromosome separation. Mol Biol Cell 25, 606-619.

44. Seldin, L., Poulson, N.D., Foote, H.P., and Lechler, T. (2013). NuMA localization, stability, and function in spindle orientation involve 4.1 and Cdk1 interactions. Mol Biol Cell 24, 3651-3662.

45. Kotak, S., Busso, C., and Gonczy, P. (2014). NuMA interacts with 
phosphoinositides and links the mitotic spindle with the plasma membrane. Embo J.

46. Compton, D.A., and Luo, C. (1995). Mutation of the predicted p34cdc2 phosphorylation sites in NuMA impair the assembly of the mitotic spindle and block mitosis. J Cell Sci 108 ( Pt 2), 621-633.

47. Kotak, S., Busso, C., and Gonczy, P. (2013). NuMA phosphorylation by CDK1 couples mitotic progression with cortical dynein function. Embo $\mathrm{J}$ 32, 2517-2529.

48. Baines, A.J., Lu, H.C., and Bennett, P.M. (2014). The Protein 4.1 family: hub proteins in animals for organizing membrane proteins. Biochim Biophys Acta 1838, 605-619.

49. Mattagajasingh, S.N., Huang, S.C., Hartenstein, J.S., Snyder, M., Marchesi, V.T., and Benz, E.J. (1999). A nonerythroid isoform of protein $4.1 \mathrm{R}$ interacts with the nuclear mitotic apparatus (NuMA) protein. J Cell Biol 145, 29-43.

50. Poulson, N.D., and Lechler, T. (2010). Robust control of mitotic spindle orientation in the developing epidermis. J Cell Biol 191, 915-922.

51. Segalen, M., Johnston, C.A., Martin, C.A., Dumortier, J.G., Prehoda, K.E., David, N.B., Doe, C.Q., and Bellaiche, Y. (2010). The Fz-Dsh planar cell polarity pathway induces oriented cell division via Mud/NuMA in Drosophila and zebrafish. Dev Cell 19, 740-752.

52. Lancaster, O.M., Le Berre, M., Dimitracopoulos, A., Bonazzi, D., Zlotek-Zlotkiewicz, E., Picone, R., Duke, T., Piel, M., and Baum, B. (2013). Mitotic rounding alters cell geometry to ensure efficient bipolar spindle formation. Dev Cell 25, 270-283.

53. Cadart, C., Zlotek-Zlotkiewicz, E., Le Berre, M., Piel, M., and Matthews, H.K. (2014). Exploring the function of cell shape and size during mitosis. Dev Cell 29, 159-169.

54. Nachury, M.V., Maresca, T.J., Salmon, W.C., Waterman-Storer, C.M., Heald, R., and Weis, K. (2001). Importin beta is a mitotic target of the small GTPase Ran in spindle assembly. Cell 104, 95-106.

55. Wiese, C., Wilde, A., Moore, M.S., Adam, S.A., Merdes, A., and Zheng, Y. (2001). Role of importin-beta in coupling Ran to downstream targets in 
microtubule assembly. Science 291, 653-656.

56. Matsumura, S., Hamasaki, M., Yamamoto, T., Ebisuya, M., Sato, M., Nishida, E., and Toyoshima, F. (2012). ABL1 regulates spindle orientation in adherent cells and mammalian skin. Nat Commun 3, 626.

57. Collins, E.S., Balchand, S.K., Faraci, J.L., Wadsworth, P., and Lee, W.L. (2012). Cell cycle-regulated cortical dynein/dynactin promotes symmetric cell division by differential pole motion in anaphase. Mol Biol Cell 23, 3380-3390.

58. Liu, D., Vader, G., Vromans, M.J., Lampson, M.A., and Lens, S.M. (2009). Sensing chromosome bi-orientation by spatial separation of aurora $B$ kinase from kinetochore substrates. Science 323, 1350-1353.

59. Welburn, J.P., Vleugel, M., Liu, D., Yates, J.R., 3rd, Lampson, M.A., Fukagawa, T., and Cheeseman, I.M. (2010). Aurora B phosphorylates spatially distinct targets to differentially regulate the kinetochore-microtubule interface. Mol Cell 38, 383-392.

60. Padte, N.N., Martin, S.G., Howard, M., and Chang, F. (2006). The cell-end factor pom $1 p$ inhibits mid1p in specification of the cell division plane in fission yeast. Curr Biol 16, 2480-2487.

61. Moseley, J.B., Mayeux, A., Paoletti, A., and Nurse, P. (2009). A spatial gradient coordinates cell size and mitotic entry in fission yeast. Nature 459, 857-860.

62. Martin, S.G., and Berthelot-Grosjean, M. (2009). Polar gradients of the DYRK-family kinase Pom1 couple cell length with the cell cycle. Nature 459, 852-856.

63. Howard, M. (2012). How to build a robust intracellular concentration gradient. Trends Cell Biol 22, 311-317.

64. Kiekebusch, D., and Thanbichler, M. (2014). Spatiotemporal organization of microbial cells by protein concentration gradients. Trends in microbiology 22, 65-73.

65. Dogterom, M., Kerssemakers, J.W., Romet-Lemonne, G., and Janson, M.E. (2005). Force generation by dynamic microtubules. Curr Opin Cell Biol 17, 67-74.

66. Goshima, G., and Scholey, J.M. (2010). Control of mitotic spindle length. 
Annu Rev Cell Dev Biol 26, 21-57.

67. Fink, J., Carpi, N., Betz, T., Betard, A., Chebah, M., Azioune, A., Bornens, M., Sykes, C., Fetler, L., Cuvelier, D., et al. (2011). External forces control mitotic spindle positioning. Nat Cell Biol 13, 771-778.

68. London, N., and Biggins, S. (2014). Signalling dynamics in the spindle checkpoint response. Nat Rev Mol Cell Biol 15, 736-748.

69. Xiao, Z., Wan, Q., Du, Q., and Zheng, Z. (2012). Galpha/LGN-mediated asymmetric spindle positioning does not lead to unequal cleavage of the mother cell in 3-D cultured MDCK cells. Biochem Biophys Res Commun 420, 888-894.

70. Sedzinski, J., Biro, M., Oswald, A., Tinevez, J.Y., Salbreux, G., and Paluch, E. (2011). Polar actomyosin contractility destabilizes the position of the cytokinetic furrow. Nature 476, 462-466.

71. Salbreux, G., Charras, G., and Paluch, E. (2012). Actin cortex mechanics and cellular morphogenesis. Trends Cell Biol 22, 536-545.

72. Connell, M., Cabernard, C., Ricketson, D., Doe, C.Q., and Prehoda, K.E. (2011). Asymmetric cortical extension shifts cleavage furrow position in Drosophila neuroblasts. Mol Biol Cell 22, 4220-4226.

73. Kunda, P., Rodrigues, N.T., Moeendarbary, E., Liu, T., Ivetic, A., Charras, G., and Baum, B. (2012). PP1-mediated moesin dephosphorylation couples polar relaxation to mitotic exit. Curr Biol 22, 231-236.

74. Roubinet, C., Decelle, B., Chicanne, G., Dorn, J.F., Payrastre, B., Payre, F., and Carreno, S. (2011). Molecular networks linked by Moesin drive remodeling of the cell cortex during mitosis. J Cell Biol 195, 99-112.

75. D'Avino, P.P. (2009). How to scaffold the contractile ring for a safe cytokinesis - lessons from Anillin-related proteins. J Cell Sci 122, 1071-1079.

76. Silverman-Gavrila, R.V., Hales, K.G., and Wilde, A. (2008). Anillin-mediated targeting of peanut to pseudocleavage furrows is regulated by the GTPase Ran. Mol Biol Cell 19, 3735-3744.

77. Archambault, V., and Glover, D.M. (2009). Polo-like kinases: conservation and divergence in their functions and regulation. Nat Rev Mol Cell Biol 10, 265-275. 
78. Petronczki, M., Lenart, P., and Peters, J.M. (2008). Polo on the Rise-from Mitotic Entry to Cytokinesis with Plk1. Dev Cell 14, 646-659.

79. Kalab, P., and Heald, R. (2008). The RanGTP gradient - a GPS for the mitotic spindle. J Cell Sci 121, 1577-1586.

80. Hasegawa, K., Brenner, B.E., Clark, S., and Camargo, C.A. (2014). Emergency department visits for acute asthma by adults who ran out of their inhaled medications. Allergy Asthma Proc 35, 42-50.

81. Deng, M., Suraneni, P., Schultz, R.M., and Li, R. (2007). The Ran GTPase mediates chromatin signaling to control cortical polarity during polar body extrusion in mouse oocytes. Dev Cell 12, 301-308.

82. Rivers, D.M., Moreno, S., Abraham, M., and Ahringer, J. (2008). PAR proteins direct asymmetry of the cell cycle regulators Polo-like kinase and Cdc25. J Cell Biol 180, 877-885.

83. Budirahardja, Y., and Gonczy, P. (2008). PLK-1 asymmetry contributes to asynchronous cell division of C. elegans embryos. Development 135, 1303-1313.

84. Cabernard, C., Prehoda, K.E., and Doe, C.Q. (2010). A spindle-independent cleavage furrow positioning pathway. Nature 467, 91-94.

85. Izumi, Y., Ohta, N., Hisata, K., Raabe, T., and Matsuzaki, F. (2006). Drosophila Pins-binding protein Mud regulates spindle-polarity coupling and centrosome organization. Nat Cell Biol 8, 586-593.

86. Siller, K.H., Cabernard, C., and Doe, C.Q. (2006). The NuMA-related Mud protein binds Pins and regulates spindle orientation in Drosophila neuroblasts. Nat Cell Biol 8, 594-600.

87. Bowman, S.K., Neumuller, R.A., Novatchkova, M., Du, Q., and Knoblich, J.A. (2006). The Drosophila NuMA Homolog Mud regulates spindle orientation in asymmetric cell division. Dev Cell 10, 731-742.

88. Cai, Y., Yu, F., Lin, S., Chia, W., and Yang, X. (2003). Apical complex genes control mitotic spindle geometry and relative size of daughter cells in Drosophila neuroblast and pl asymmetric divisions. Cell 112, 51-62.

89. Albertson, R., and Doe, C.Q. (2003). Dlg, Scrib and Lgl regulate neuroblast cell size and mitotic spindle asymmetry. Nat Cell Biol 5, 
166-170.

90. Basto, R., Lau, J., Vinogradova, T., Gardiol, A., Woods, C.G., Khodjakov, A., and Raff, J.W. (2006). Flies without centrioles. Cell 125, 1375-1386.

91. Izumi, Y., Ohta, N., Itoh-Furuya, A., Fuse, N., and Matsuzaki, F. (2004). Differential functions of $G$ protein and Baz-aPKC signaling pathways in Drosophila neuroblast asymmetric division. J Cell Biol 164, 729-738.

92. Cheeseman, I.M. (2014). The Kinetochore. Cold Spring Harb Perspect Biol 6.

93. Laan, L., Pavin, N., Husson, J., Romet-Lemonne, G., van Duijn, M., Lopez, M.P., Vale, R.D., Julicher, F., Reck-Peterson, S.L., and Dogterom, M. (2012). Cortical dynein controls microtubule dynamics to generate pulling forces that position microtubule asters. Cell 148, 502-514.

94. Schmidt, J.C., Arthanari, H., Boeszoermenyi, A., Dashkevich, N.M., Wilson-Kubalek, E.M., Monnier, N., Markus, M., Oberer, M., Milligan, R.A., Bathe, M., et al. (2012). The kinetochore-bound Ska1 complex tracks depolymerizing microtubules and binds to curved protofilaments. Dev Cell 23, 968-980.

95. Trokter, M., Mucke, N., and Surrey, T. (2012). Reconstitution of the human cytoplasmic dynein complex. Proc Natl Acad Sci U S A 109, 20895-20900.

96. Schlager, M.A., Hoang, H.T., Urnavicius, L., Bullock, S.L., and Carter, A.P. (2014). In vitro reconstitution of a highly processive recombinant human dynein complex. Embo J 33, 1855-1868.

97. McKenney, R.J., Huynh, W., Tanenbaum, M.E., Bhabha, G., and Vale, R.D. (2014). Activation of cytoplasmic dynein motility by dynactin-cargo adapter complexes. Science 345, 337-341.

98. McKenney, R.J., Vershinin, M., Kunwar, A., Vallee, R.B., and Gross, S.P. (2010). LIS1 and NudE induce a persistent dynein force-producing state. Cell 141, 304-314.

99. Huang, J., Roberts, A.J., Leschziner, A.E., and Reck-Peterson, S.L. (2012). Lis1 acts as a "clutch" between the ATPase and microtubule-binding domains of the dynein motor. Cell 150, 975-986.

100. Chew, T.G., Lorthongpanich, C., Ang, W.X., Knowles, B.B., and Solter, D. 
(2012). Symmetric cell division of the mouse zygote requires an actin network. Cytoskeleton (Hoboken) 69, 1040-1046. 
(a)

\begin{tabular}{c}
$\begin{array}{c}\text { Spindle Orientation } \\
\text { Control }\end{array}$ \\
\hline
\end{tabular}

Polarized factor
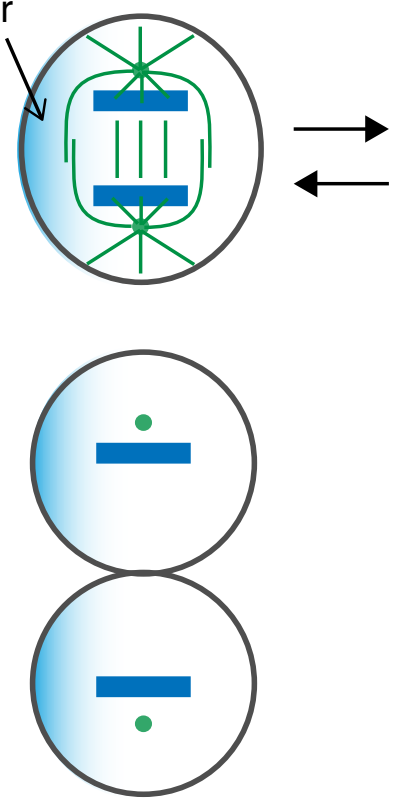

(b)

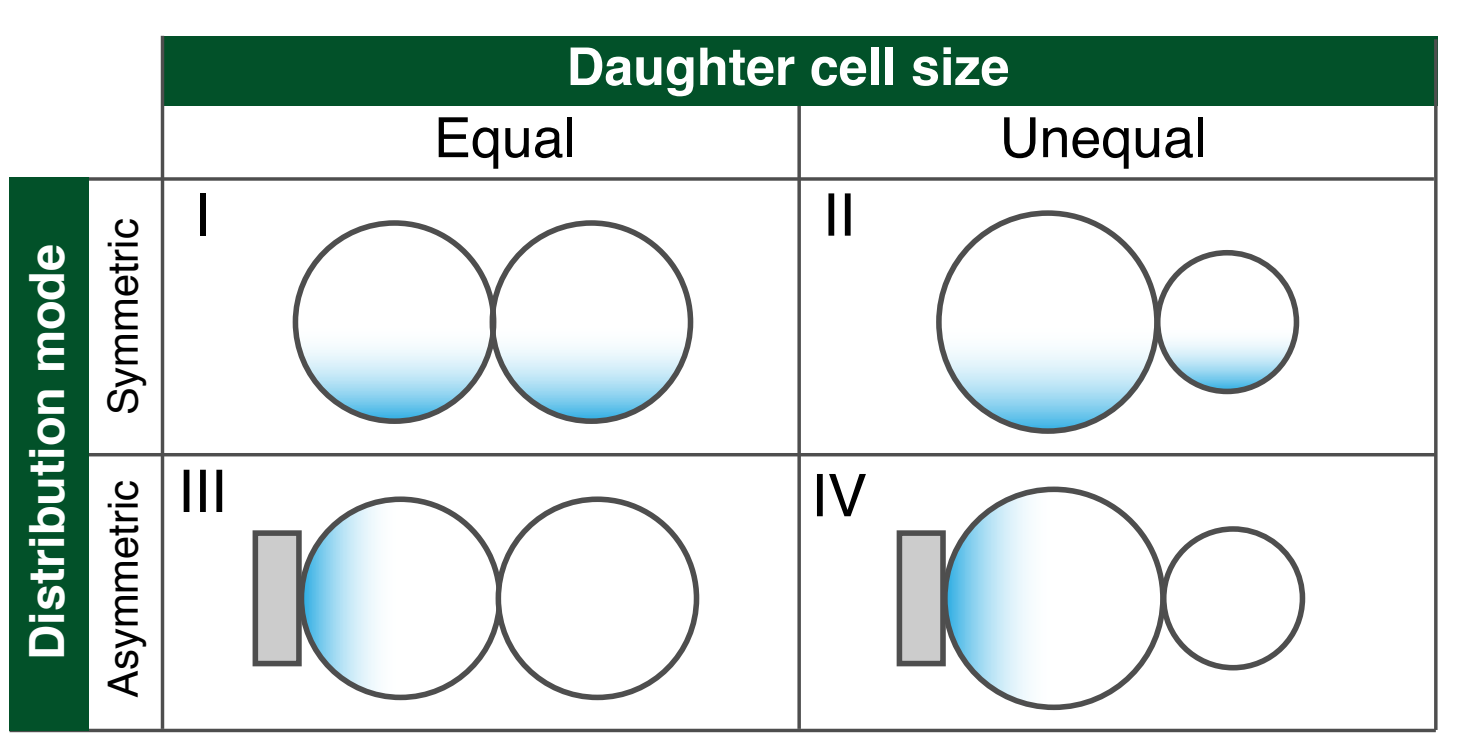

Intrinsic ( $($ ) or extrinsic ( $\square$ ) polarized factors
Cleavage site

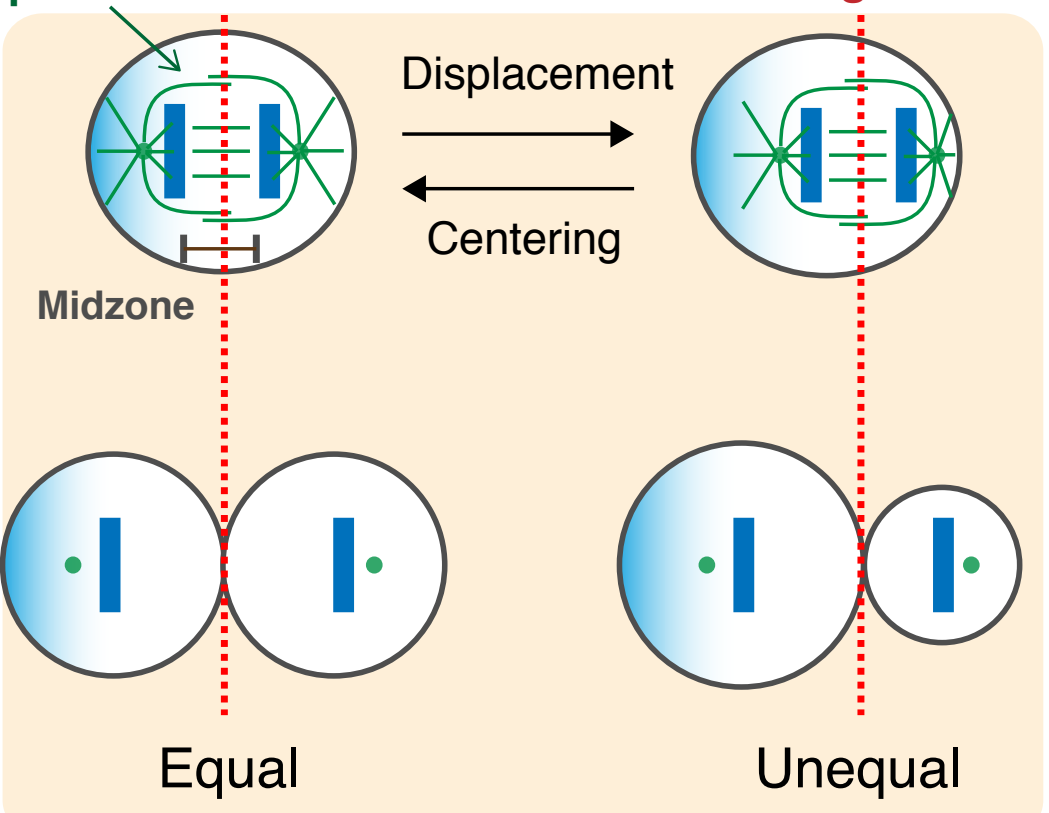

Equal
) 
Symmetric cortical pulling force

(a) Unequally dividing C.elegans embryos

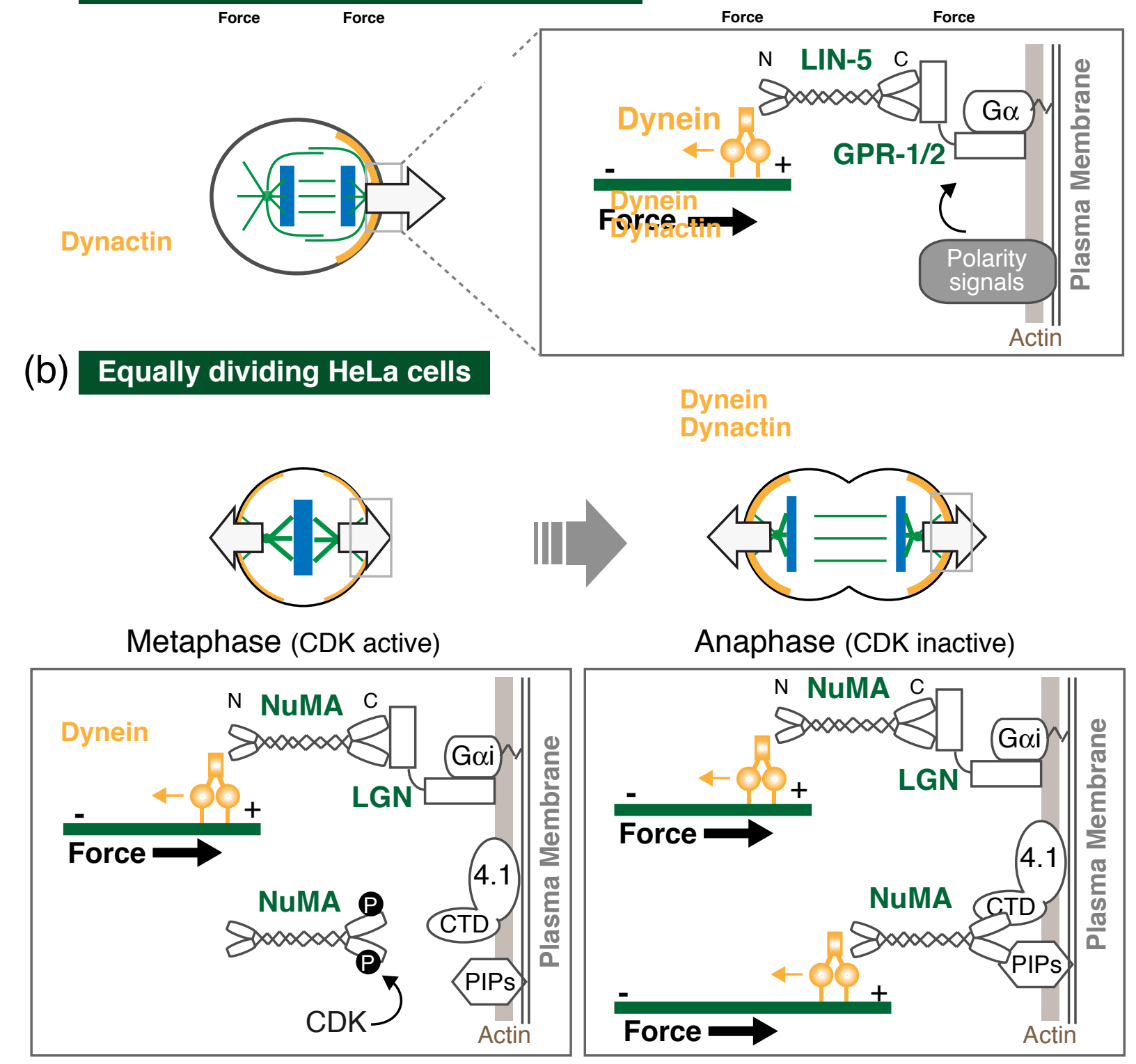


Fig.3

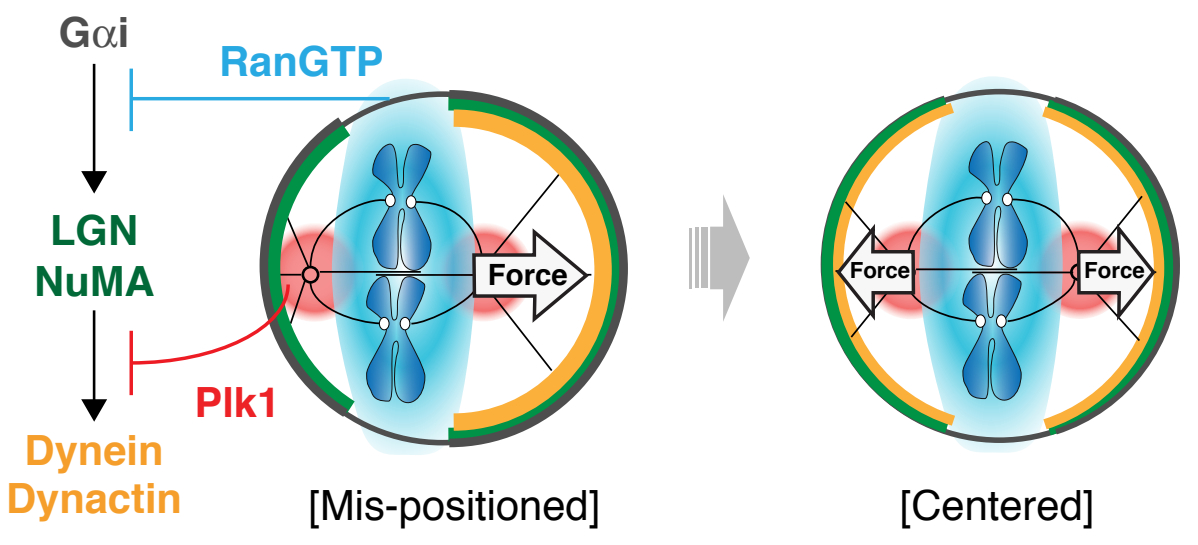


(a)

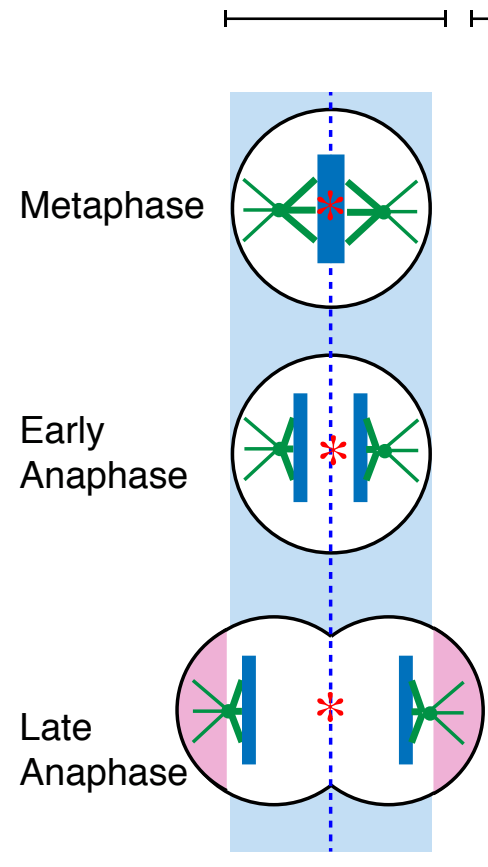

(b)

\section{(Polar Membrane)}

PIPs 4.1 Goi

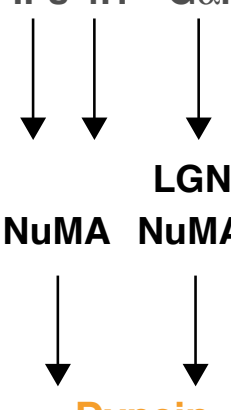

Dynein

(Pulling force)
1. Move the spindle

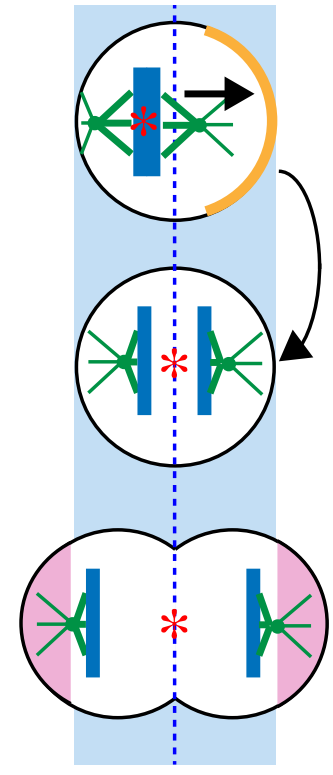

2. Alter cellular boundary

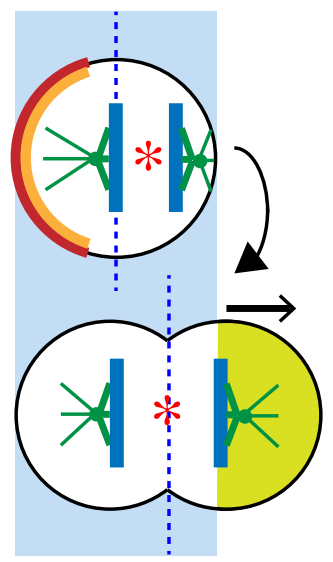

(c)

(Polar Membrane)

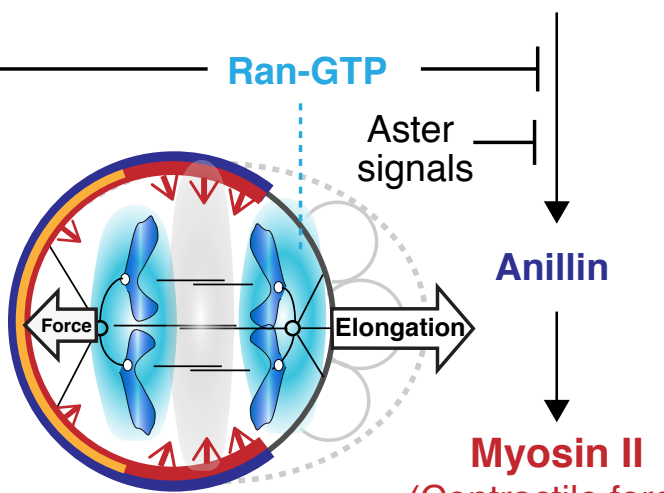

(Contractile force)

\begin{tabular}{|cc|}
\hline * Center of the spindle & $\vdots$ \\
Dynein asymmetry & Cell center \\
Myosin asymmetry
\end{tabular}

3.Elongate the spindle

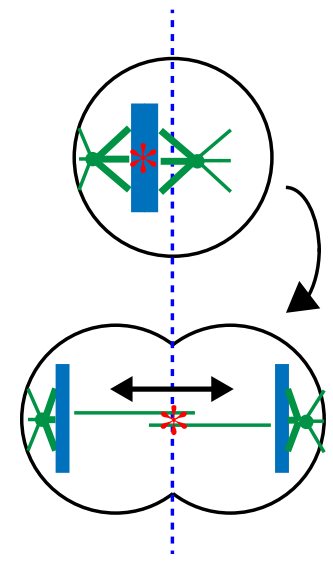

Forces that alter cellular boundary Symmetric

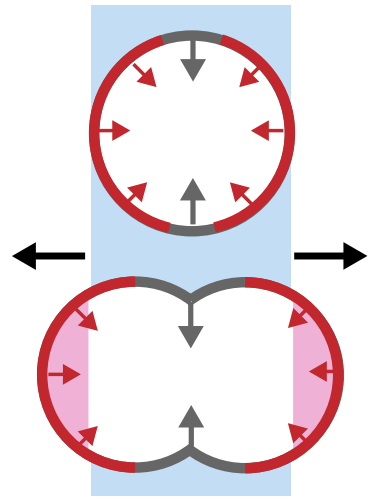

Asymmetric

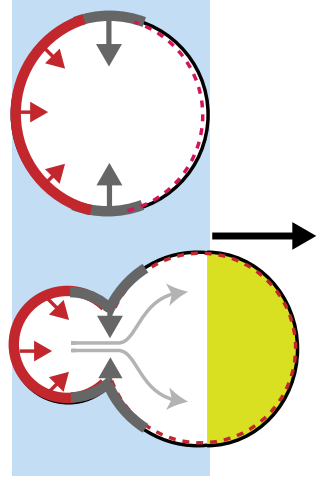


(a)

\section{Equal-sized cell division}

Nucleus and/or

spindle centering

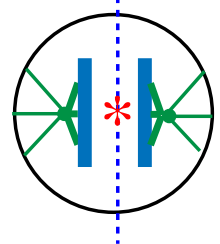

[Centered]

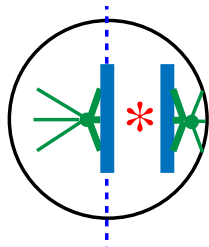

[Mis-positioned]
Symmetric

force generation

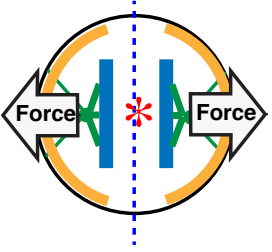

Asymmetric force generation
Cortical Dynein Polar Myosin Equal-sized daughters

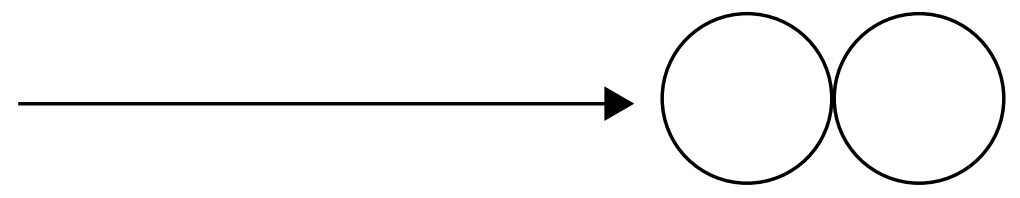

Spindle centering (Forces are balanced)

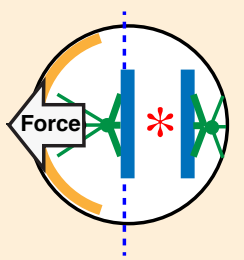

Move the spindle
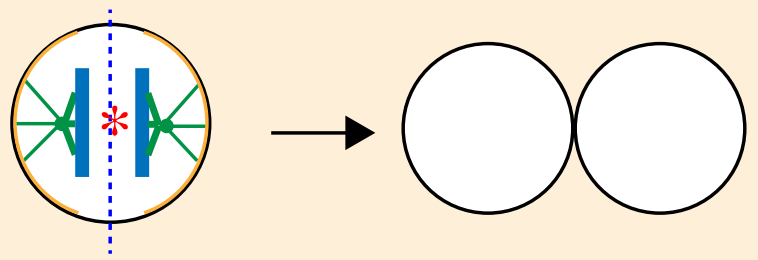

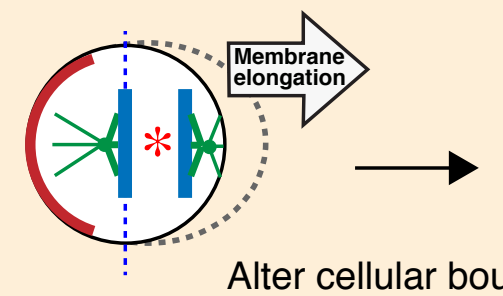

Alter cellular boundary
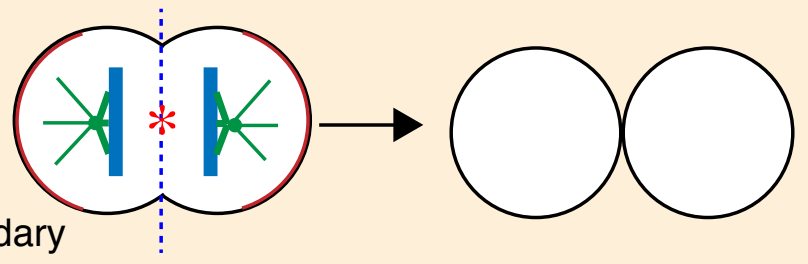

\section{(b) Unequal-sized cell division}

Nucleus and/or spindle centering

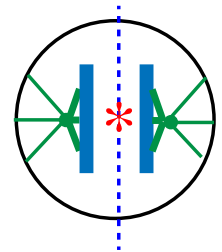

[Centered]
Asymmetric force generation
Spindle displacement (Forces are still asymmetric)
Unequal-sized daughters
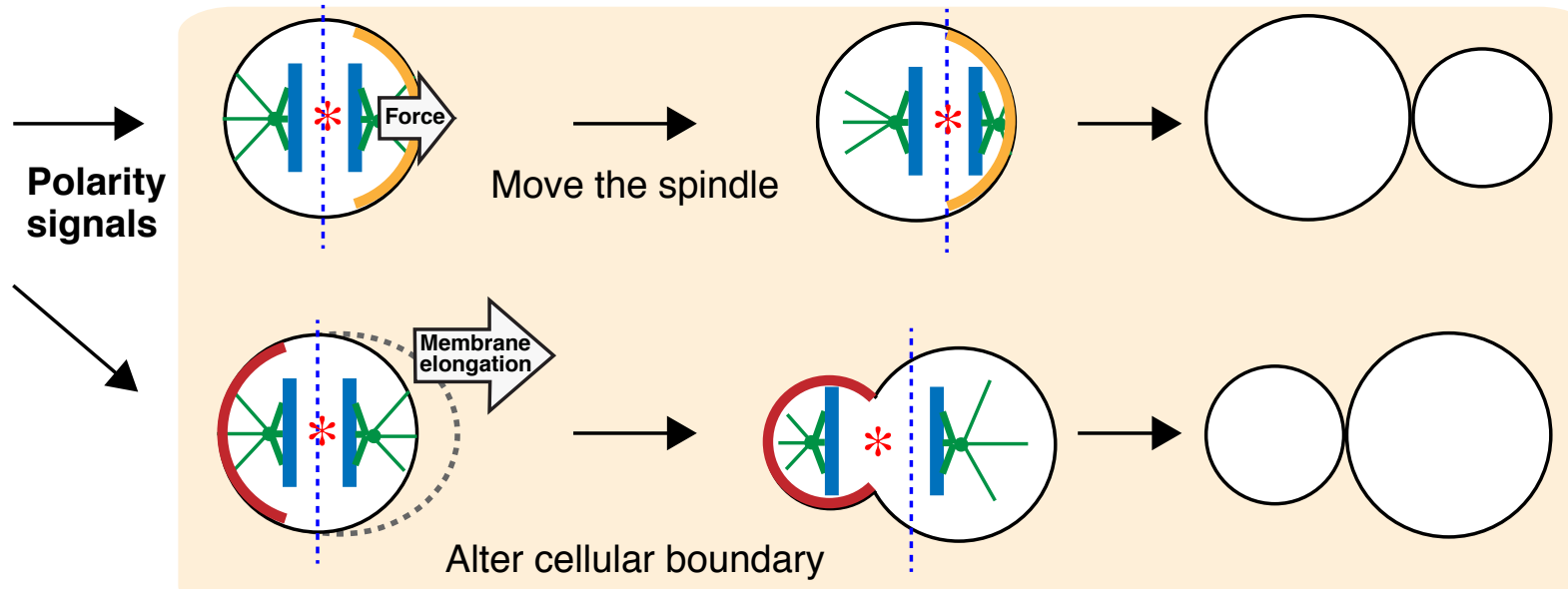